\title{
Management of meningiomas involving the transverse or sigmoid sinus
}

\author{
Marcus D. Mazur, M.D., Aaron Cutler, M.D., William T. Couldwell, M.D., Ph.D., \\ And Philipp Taussky, M.D.
}

Department of Neurosurgery, Clinical Neurosciences Center, University of Utah, Salt Lake City, Utah

\begin{abstract}
Meningiomas that invade the transverse or sigmoid sinuses are uncommon tumors that are challenging to treat surgically. Although the risk of recurrence is associated with the extent of resection, complete removal of meningiomas in these locations must be balanced with avoidance of venous outflow obstruction, which could cause venous infarction and significant neurological consequences. When a meningioma occludes a venous sinus completely, grosstotal resection of the intravascular portion is commonly performed. When the tumor invades but does not completely obliterate a major venous sinus, however, opinions differ on whether to accept a subtotal resection or to open the sinus, perform a complete resection, and reconstruct the venous outflow tract. In this paper, the authors review the different strategies used to treat these lesions and provide illustrative case examples.

(http://thejns.org/doi/abs/10.3171/2013.8.FOCUS13340)
\end{abstract}

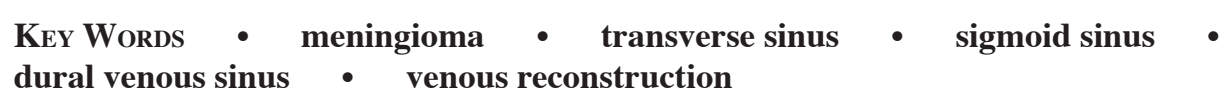

$\mathrm{M}$ ENINGIOMAs arise from arachnoid cap cells in arachnoid granulations and often invade large veins and venous sinuses. At the transverse sinus, transverse-sigmoid sinus junction, and peritorcular region, arachnoid cap cells are particularly abundant, ${ }^{6}$ yet meningiomas with dural attachments at the region of the transverse-sigmoid sinus junction are relatively uncommon. While $7 \%-10 \%$ of meningiomas are located in the posterior fossa, only $10 \%$ of these involve the transverse or sigmoid sinus. ${ }^{28}$ Of all meningiomas involving the major dural sinuses, only $5 \%$ occur at the transverse sinus. ${ }^{43}$

Patients who have meningiomas at the transverse or sigmoid sinuses may present with signs of mass effect, including headache, dizziness, papilledema, and ataxia. If a dominant sinus is occluded or severely stenotic, the patient may experience increased intracranial pressure from impaired venous drainage. ${ }^{42}$ Authors have reported cases of dural arteriovenous fistulas arising from venous sinus flow compromise due to tumor obstruction., ${ }^{2,50}$ Often, however, considering that most meningiomas are slowgrowing lesions, patients may remain asymptomatic for a long period because tumors in this region can grow very large and spread to multiple compartments before they are diagnosed. Some meningiomas may extend into the

Abbreviations used in this paper: GTR = gross-total resection; $\mathrm{WHO}=$ World Health Organization . vein of Labbé, ${ }^{17}$ which connects the superficial sylvian vein to the transverse sinus in proximity to the transversesigmoid junction and provides the main outflow pathway for the inferior cerebral venous system. Occlusion of this vein may produce severe complications, such as hemorrhagic infarction of the temporal lobe, cerebral edema, neurological dysfunction, seizures, or even death. If the lateral temporal lobe lacks sufficient collateral venous drainage, the consequences of vein of Labbé occlusion can be devastating.

Radical resection of meningiomas that invade the major dural sinuses is controversial and often limited by the tumor's relationship with venous circulation. When the venous sinuses are preserved, resection of meningiomas at the transverse-sigmoid junction carries a relatively low surgical risk. If the sinuses are invaded, many authors advocate complete resection of the intrasinus portions of the tumor, with vessel reconstruction, if necessary, to prevent recurrence. ${ }^{41,43}$ But this technique must be balanced with the avoidance of venous outflow obstruction, which could cause venous infarction and significant neurological consequences. When a meningioma occludes a venous sinus completely, gross-total resection (GTR) of the intravascular portion is commonly performed. When the tumor invades but does not completely obliterate a major venous sinus, however, opinions differ on whether to accept a subtotal resection or to open the sinus, perform a complete resection, and then reconstruct the venous outflow tract. 


\section{Simpson Grading}

In 1957, Simpson $^{40}$ reported that the likelihood of meningioma recurrence decreases with greater degrees of resection: Complete tumor removal with excision of its dural attachment and removal of the intravascular portion had a lower rate of recurrence (Simpson Grade I, 9\% recurrence) than tumor removal with only coagulation of the dura mater (Grade II, 19\% recurrence), resection of the tumor itself but without treatment of the dural attachment or intravascular portion (Grade III, 29\% recurrence), or subtotal resections (Grade IV, 39\% recurrence). However, some recent reports have indicated that recurrence rates are similar among Simpson grades and that the risk of additional morbidity does not warrant resecting remnant tumor around critical structures, such as within the dural sinuses, to achieve a higher Simpson grade..$^{20,31,46}$ This trend likely reflects technological advances that enable modern surgeons to resect tumors more aggressively so that the portions adherent to cranial nerves and blood vessels that are left behind are smaller than in the past. Nevertheless, such studies do not provide clear evidence regarding long-term outcomes on the extent of resection and meningioma recurrence, and many surgeons still advocate complete or near-total resection, ${ }^{43}$ particularly in younger patients and when additional resection does not carry significant risk..$^{19,31}$

\section{Surgical Planning}

Initial evaluation of a meningioma involving the transverse sinus should include a determination of the extent of intravascular tumor invasion, the degree of sinus occlusion, and the anatomy of venous outflow, including the presence of large draining veins, aberrant drainage, and collateral vessels. Various classification schemes of meningiomas involving the dural venous sinuses have been published, most of which classify tumors according to the degree of sinus wall involvement. ${ }^{4,43}$ Mantovani et al. ${ }^{29}$ proposed a more clinically relevant system that considers the degree of sinus patency: total occlusion, subtotal with 50\%-95\% occlusion, and partial with less than $50 \%$ occlusion. Patients with subtotal or partial sinus occlusions may have different risk profiles than those with complete occlusion, and this may influence decision making regarding venous reconstruction.

In most cases, MR or CT venous angiography is sufficient for initial evaluation of venous occlusion. If the tumor has intravascular extension, conventional angiography may be helpful in differentiating severe luminal narrowing versus complete sinus occlusion, which would be particularly useful in the setting of a dominant, aberrant, or unilateral transverse sinus. ${ }^{23,28}$ In such cases, many surgeons would elect to preserve existing venous drainage and limit resection to the extravascular portions of the tumor with the goal of leaving current venous outflow intact. Angiography may help to identify aberrant drainage caused by progressive sinus occlusion, such as flow-related dural arteriovenous fistulas or venous collateralization. If the sinus is partially occluded, care must be taken to avoid interrupting its flow, since this may precipitate brain swelling, venous infarction, and CSF obstruction. This is especially true if the degree of occlusion is mild because collateralization may not have had enough time to develop. ${ }^{38}$ Radiographic evidence of sinus occlusion should be interpreted cautiously. In some cases, the tumor may only partially occlude the sinus or alter flow in such a way that the contrast-enhanced and/or vascular imaging protocols do not accurately detect vessel patency. Surgeons should be prepared for brisk venous bleeding whenever the sinus is opened. If the sinus appears occluded radiographically but flow is identified at surgery, care must be taken to repair the vessel after tumor removal to avoid disrupting venous outflow. In such cases, we use a monofilament suture (Proline, 6-0 or 7-0) to reduce thrombogenicity.

Resecting a completely occluded portion of the sinus is a matter of current debate. Although historically this practice was generally accepted and performed routinely, studies on parasagittal meningiomas involving the superior sagittal sinus have shown that resection of occluded portions can interrupt collateral venous drainage, which may lead to venous hypertension and its sequelae in some patients if venous flow is not restored. ${ }^{4,13,15,21,43}$ While few studies have documented outcomes after resection of occluded portions of the transverse sinus, similar morbidity would likely occur if collateral flow is disrupted, affecting veins corresponding to the inferior cerebral veins of Labbé. Therefore, venous reconstruction may be useful to restore outflow of the compensatory collateral channels that may have developed as the intraluminal tumor obstructed venous drainage. ${ }^{43}$

Histological grading is an important factor to be considered when planning a treatment strategy. World Health Organization (WHO) Grade II and III meningiomas demonstrate much higher recurrence rates and tumor-associated morbidity and mortality. Atypical meningiomas carry a 7 - to 8 -fold increased risk of recurrence and a 2 -fold increased risk of death at 3-5 years after resection. ${ }^{1,36}$ It is generally accepted that complete removal of these lesions, with or without radiation therapy, is important for survival. Intraoperative frozen section pathology can be performed early during tumor removal to help guide the extent of resection. ${ }^{29}$ Meningiomas with aggressive features or high histological grade are then treated more aggressively, while those that appear benign are treated conservatively.

\section{Surgical Technique}

The management of meningiomas invading the sinuses can be divided into strategies based on the extent of sinus manipulation. Conservative management consists of debulking a tumor from the outer walls of the sinus, carefully removing it from the brain, and resecting any involved portions of the tentorium. The dissection is extravascular, the sinus is not opened, and remnant tumor is treated with adjuvant radiation therapy or is observed. Aggressive management with a goal of GTR of the meningioma involves opening the sinus, resecting the intravascular portion of the tumor, and performing venous reconstruction or ligation. 
If the decision to open the sinus will be made intraoperatively, the patient is counseled of this possibility beforehand. The patient undergoes neuromonitoring with motor evoked potentials and somatosensory evoked potentials. A precordial Doppler probe and a central venous catheter are placed to detect a possible air embolism. An external ventricular drain can be placed for intracranial pressure monitoring and CSF diversion. If sinus repair is planned, a preoperative dose of aspirin is administered. Attention must be paid to the position of the head in relation to the heart. If the head is at or above the heart, an air embolism during sinus intervention is a real possibility. We prefer to have the head low enough so that venous bleeding is readily detectable and the risk of air embolism is reduced. Theoretically, the high intracranial venous pressure in these patients decreases the chance of an air embolism. ${ }^{41}$ The surgical exposure should be wide, meaning that the craniotomy should extend across both sides of the sinus and well past the distal margin of the occluded portions. Bridging veins should be dissected free and preserved.

\section{Aggressive Management}

Once a decision is made to open the sinus, the surgical strategy depends on the extent of sinus occlusion. If the sinus is completely occluded, the involved segment can be removed and the vessel reconstructed. The sinus is opened at the region of tumor invasion, and the meningioma is removed from the occluded central portion toward each free side. ${ }^{29}$ Care is taken to preserve any normal, tumor-free sinus walls, as well as the ostia of affluent veins. When venous bleeding is encountered from the normal ends of the sinus, temporary hemostasis is obtained. Sindou and Alvernia ${ }^{43}$ reported good results in packing small pledgets of Surgicel (Johnson Medical) into the vessel orifice, since septations in the sinus lumen can obstruct the passage of balloons and shunts, and vascular clamps and aneurysm clips can crush the sinus walls. This process is then repeated at the other free end. The pledgets of Surgicel are removed, and the sinus is reconstructed by sewing in a dural flap or patch with a monofilament suture (Proline, 6-0 or 7-0). Flow is verified using a Doppler probe or indocyanine green fluorescence angiography.

For long segments of sinus occlusion not amenable to reconstruction, the transverse sinus can be ligated in certain situations. This decision should be made judiciously because of the risk for venous infarction if ligation obstructs the outflow of collateral vessels. Ligation should only be performed if the contralateral sinus is patent or collateral venous drainage is adequate. ${ }^{23}$ Some surgeons advocate intraoperative test clamping with a Yasargil temporary clip and monitoring the surrounding brain for swelling before ligating the sinuses. ${ }^{12,23}$ Others describe measuring the sinus pressure after temporary occlusion to assess contralateral venous drainage before performing the ligation or reconstruction..$^{29,37,45}$ This is done by inserting a small-caliber needle connected to a manometer or arterial pressure monitoring line. If there is adequate drainage via the contralateral transverse sinus, intravascular pressure should increase no more than $7 \mathrm{~mm} \mathrm{Hg}$ after occlusion of the sinus. If the intravascular pressure increases more than $10 \mathrm{~mm} \mathrm{Hg}$, the sinus should be kept intact. ${ }^{45}$ If the transverse sinus is to be sacrificed, ligation is performed proximal to the superior petrosal sinus and distal to the vein of Labbé. The sigmoid sinus can be sacrificed at any point distal to the superior petrosal sinus and vein of Labbé. Once the sigmoid sinus is sacrificed, the ipsilateral vein of Labbé drains contralaterally since it enters the lateral sinus above the junction of the superior petrosal and sigmoid sinuses. ${ }^{45}$

If the sinus is partially occluded, preserving its patency is important. Normal vessel walls should be kept intact whenever possible. After the intraluminal portion of tumor is removed, the sinus wall can be repaired via resuturing, patching, or bypass. The proximal end of the sinus is allowed to bleed back into the surgical field during repair to minimize thrombosis. ${ }^{29}$ If the meningioma involves the confluence of sinuses or dominant transverse sinus, an intraluminal shunt can be used. ${ }^{29}$ Patching materials include dura, pericranium, fascia, or synthetic graft material (for example, AlloDerm). ${ }^{17,29,43}$ We prefer to use autologous materials, and patients are placed on antiplatelet regimens postoperatively. A bypass graft can be obtained from autologous vein harvesting of either the internal saphenous or external jugular vein. ${ }^{37,39}$ Synthetic bypass grafts from Gore-Tex tubes can carry a high thrombosis rate and are generally not recommended. ${ }^{43}$ It is important to ensure that the lumen of the reconstructed vessel remains of sufficient size. If sinus repair results in a smaller vessel lumen, thrombosis may develop or patients may have insufficient venous return. Successful reconstruction of the vein of Labbé has also been described. ${ }^{17}$

Some authors are in favor of flow restoration with sinus reconstruction or bypass even when there is complete sinus occlusion. ${ }^{41,43}$ Since the bypass graft allows time for compensatory collateral venous pathways to form, patients may be more likely to tolerate alterations to the venous circulations that may occur after resection. Delayed complications and deaths due to brain swelling have been observed in patients who did not undergo venous reconstruction, with meningiomas occluding both walls of the sinus. ${ }^{43}$

Resecting the portion of sinus that is occluded by tumor is not without risks. At times, the surgical approach may require sacrificing collateral veins to gain access to the occluded section of sinus. ${ }^{41}$ Such additional disruption of venous drainage in the setting of venous sinus occlusion can exacerbate brain swelling and manifest as postoperative edema. ${ }^{24,48}$

Stent placement in the venous sinus has been described as a minimally invasive technique for managing focal venous sinus thrombosis. The role of transverse sinus obstruction and the development of increased intracranial hypertension are well documented. ${ }^{11,14,25}$ Successful resolution of papilledema from idiopathic intracranial hypertension associated with focal transverse sinus thrombosis has been reported. ${ }^{30}$ Similarly, case reports have described stenting across the portion of sinus containing intravascular tumor to maintain venous patency and relieve intracranial pressure or symptomatic sinus stenosis., ${ }^{7,141}$ One potential advantage of stent placement is that if the tumor progresses but the sinus remains pat- 
ent, then resection of the tumor may be facilitated since the stent protects direct exposure of the sinus lumen, thereby obviating venous reconstruction and decreasing the risk of blood loss and venous infarction. ${ }^{14}$ Tumor resection in the setting of a stented venous sinus, however, may be technically feasible only if the intravascular portions of tumor involve only one wall of the sinus.

\section{Conservative Management}

Some surgeons advocate a more conservative approach to the management of meningiomas invading venous sinuses. This strategy consists of performing a subtotal resection of extravascular tumor without interrupting the sinus or sacrificing collateral veins. The goal is to surgically debulk the meningioma as safely as possible while reducing mass effect. Residual tumor is then treated with stereotactic radiation or radiotherapy initially or is monitored and irradiated only at the time of recurrence. Since fractionated radiation therapy and stereotactic radiosurgery of small meningiomas can lead to acceptable tumor control rates ${ }^{27}$ neurosurgeons may prefer to monitor residual disease and treat with radiosurgery rather than risk venous infarction and even death by attempting a GTR. Because of these risks, several authors consider the presence of sinus patency to be a contraindication to aggressive management. 5,35

Tomasello et al. ${ }^{49}$ described a technique for maximizing tumor resection with the primary goal of preserving venous sinus outflow, which they used in 67 patients. Preoperative angiography and MR venography were performed to delineate peritumoral venous drainage and sinus patency and for embolization, if feasible. Patent cortical bridging veins were preserved, and occluded veins were sacrificed if preoperative imaging demonstrated sufficient collateral flow. Intraoperative Doppler ultrasound was used to confirm sinus occlusion. Any attempt to resect large portions of tumor invading a patent sinus was avoided. The authors reported Simpson Grade I resection in $22 \%$ of patients, Grade II in $39 \%$, Grade III in $25 \%$, and Grade IV in 13\%. Ten percent of patients required retreatment with either repeated surgery or radiosurgery.

Caroli et al. ${ }^{5}$ described a management strategy in which meningiomas involving the superior sagittal sinus were completely resected if the sinus was completely obliterated by tumor and incompletely removed if the sinus was not occluded. Of 328 patients, 193 underwent complete resection. There were 49 cases of recurrence or regrowth, with a mean time of 6.8 years to recurrence or regrowth after Simpson Grade I resection and 4.7 years after Grade II or III resection. The extent of tumor removal was associated with recurrence or regrowth, but resection of an occluded sinus did not affect clinical outcome.

Bassiouni et al. ${ }^{3}$ described a series of 81 tentorial meningiomas, 20 of which invaded the dural sinuses. The authors adopted a conservative approach in which the involved sinuses were not opened, but residual tumor was coagulated on the sinus wall or resected if it involved only the outer dural leaflet. The recurrence rate was $8.6 \%$ after a mean follow-up of 5.9 years. In a study of 55 patients with parasagittal or falcine meningiomas and partial occlusion of the superior sagittal sinus who were not good candidates for radiosurgery, Sughrue et al. ${ }^{47}$ reported subtotal resection for 22 cases in which sinus involvement was extensive. Among these cases were 8 patients who underwent radiosurgery for residual disease. Two of the 22 cases developed recurrence after a mean follow-up of 7.6 years; one had a WHO Grade II and the other had a WHO Grade III meningioma.

Stereotactic radiosurgery has been used for the adjuvant treatment of residual tumors and primary treatment for small meningiomas $(<3 \mathrm{~cm})$ with patent sinuses. ${ }^{18,26,27}$ Some authors include adjuvant radiation therapy in their treatment regimen for atypical and malignant meningiomas, ${ }^{29}$ although this practice remains controversial. ${ }^{1,9,16,18,22,32,36}$ Pollock et al. ${ }^{34}$ demonstrated equipoise in recurrence rates after radiosurgery as the primary management strategy and after Simpson Grade I resection for benign meningiomas smaller than $3.5 \mathrm{~cm}$, and superiority of adjuvant radiotherapy when compared with Grade II-IV resections. On the other hand, for tumors that are large $(>3$ $\mathrm{cm}$ ), those with symptomatic mass effect, or those causing partial venous occlusion, radiosurgery may not be appropriate as the primary treatment. Irradiation of peritumoral veins can produce venous occlusive complications and resultant symptomatic edema. Venous occlusive complications may account for the higher morbidity rate after radiosurgery in meningiomas involving the sinuses. ${ }^{27,44}$ One way to decrease this risk is to use pretreatment CT or MR venography to delineate the peritumoral venous anatomy and establish dose constraints for each vascular structure. ${ }^{10}$ This would limit radiation to veins while maintaining adequate coverage of the target lesion.

\section{Surgical Outcomes}

Because meningiomas that involve the transverse or sigmoid sinus are uncommon, there are few outcome data to help guide treatment planning. Most relevant studies examine parasagittal meningiomas, which are located at the interface between the convexity and the falx and involve the superior sagittal sinus. In a study of attempted radical resection of meningiomas involving any major dural sinus, the tumor recurrence rate was $4 \%{ }^{43}$ In that series, all 4 recurrences involved the middle third of the superior sagittal sinus. In other series, recurrence rates have varied from $4 \%$ to $24 \% .^{29,43,49}$ A Swedish study evaluating long-term outcomes after a 25 -year follow-up noted a $47 \%$ recurrence rate and found that subtotal resection was correlated with increased tumor-related morbidity and mortality. ${ }^{33}$ Other recent retrospective series with a 10-year follow-up or less documented much lower rates of recurrence. ${ }^{5,13,43}$

In a review of the literature on meningiomas involving the superior sagittal sinus, Tomasello et al ${ }^{49}$ stratified study populations by treatment strategy from conservative to most radical: total resection in the setting of complete sinus obstruction or when the sinus was "only marginally involved"; resection of an intrasinus tumor requiring patching of the sinus but not venous reconstruction; and radical resection of a tumor with massive sinus involvement requiring bypass techniques to restore sinus outflow. These authors found that the recurrence rate was $6 \%-29 \%$ 
when a conservative strategy was adopted; $14 \%-19 \%$ when an intrasinus tumor was resected, requiring sinus repair but not reconstruction; and $4 \%$ when sinus outflow was restored after tumor resection. Of note, tumors involving the transverse or sigmoid sinuses were excluded in that study. Nevertheless, we would expect the rates of recurrence to be similar among tumors involving any of the dural venous sinuses for a given treatment strategy.

In a review of 30 tentorial meningiomas, Colli et al. ${ }^{8}$ reported involvement of the transverse or sigmoid sinus in 10 patients. Six patients had partial sinus occlusion and underwent resection of the involved wall with suture repair of the sinus without grafting; 3 underwent sinus resection without reconstruction for complete occlusion (details on 10th patient unclear). A total of $12.5 \%$ of the patients had a subtotal resection, and a recurrence rate of $12.5 \%$ was reported.

In a series of 100 cases of meningiomas involving the major dural sinuses, 5 of which involved the transverse sinus, Sindou and Alvernia ${ }^{43}$ reported a complication rate of $8 \%$ and a mortality rate of $3 \%$ after radical tumor removal. Gross-total resection was achieved in $93 \%$ of the cases, and the sinus was reconstructed in $45 \%$. All 3 patients who died had undergone resection for a tumor completely occluding the superior sagittal sinus but had not undergone venous reconstruction.

Mantovani et al..$^{29}$ reported on a series of 38 patients with meningiomas involving the major venous sinuses. Five lesions in these cases involved the transverse sinus and 2 involved the sigmoid sinus. The authors adopted an aggressive management strategy with an attempt at total tumor removal whenever possible, including the restoration of venous flow with primary suture or autologous patch. The uninvolved sinus wall was preserved whenever possible. Complete resection occurred in 33 of 38 patients, and there were only 2 recurrences (5.3\%) after a mean follow-up of 26 months. Only 1 patient had a major complication related to sinus reconstruction and venous damage, and there were no deaths. Sinus thrombosis developed in 3 patients but without postoperative brain swelling, hemorrhage, or infarction. Indeed, the authors stated that the presence of compensatory collateral venous circulation was known in these cases, since all patients underwent preoperative angiography to delineate venous anatomy.

\section{Illustrative Cases}

Few clinical studies focus specifically on meningiomas located at the transverse or sigmoid sinuses. While much can be surmised from the literature on parasagittal meningiomas in terms of the technical aspects of sinus repair and rates of recurrence, meningiomas invading the transverse or sigmoid sinus may present a different set of challenges. These lesions often occupy a unique location in the posterior fossa. Patients may be less tolerant of brain swelling if venous drainage is compromised. The vein of Labbé may be prone to changes in venous circulation, and the risk of morbidity and mortality may differ as a result of the regional anatomy. To our knowledge, there is no study with a sufficient sample size to adequately assess outcomes in this group of patients. Herein, we present a few case examples to illustrate how meningiomas invading the transverse or sigmoid sinuses are managed at our institution.

\section{Case 1}

A 36-year-old woman with a history of left carotid artery dissection requiring stent placement was found to have an enlarging, $1.6 \times 1.8-\mathrm{cm}$, homogeneously enhancing extraaxial lesion in the right posterior fossa. Preoperative vascular imaging demonstrated partial occlusion of the sigmoid sinus with possible tumor invasion (Fig. 1). The patient underwent a right retrosigmoid craniectomy for tumor resection. The tumor had invaded the sigmoid sinus and was removed from this region in its entirety. Sinus bleeding was controlled with Surgicel pledgets and direct pressure. Primary suture repair or grafting of the sinus was not necessary. Pathological analysis was consistent with WHO Grade I meningioma. Postoperative imaging demonstrated gross-total tumor resection with patent transverse or sigmoid sinuses (Fig. 2). Follow-up imaging at 2.5 years after resection showed no evidence of tumor recurrence.

\section{Case 2}

A 69-year-old man with minor head trauma underwent imaging that showed a $4 \times 3 \times 4-\mathrm{cm}$ homogeneously enhancing, right tentorial extraaxial lesion with involvement of the right transverse sinus (Fig. 3A and B). A right
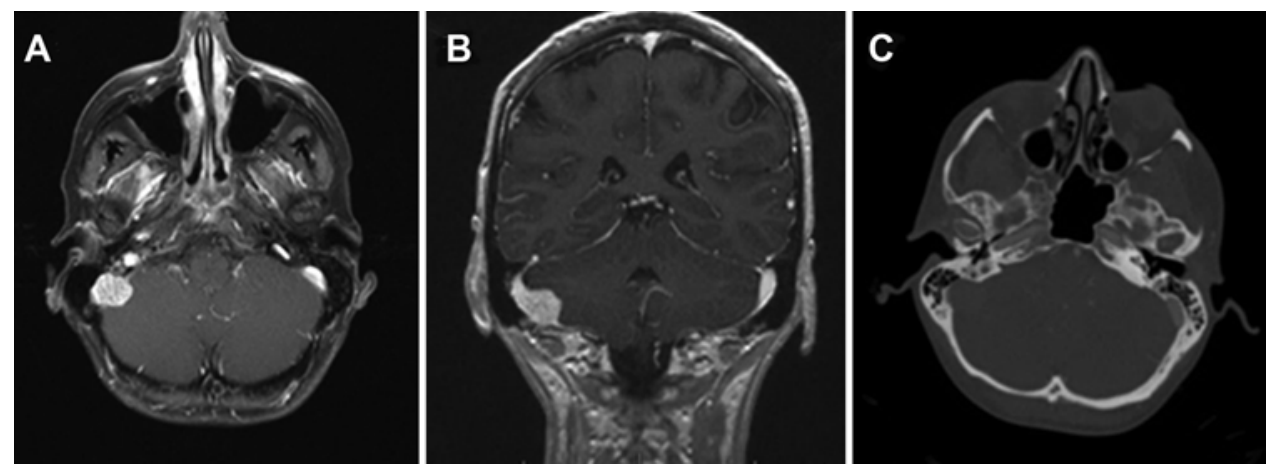

FIG. 1. Case 1. Preoperative axial (A) and coronal (B) T1-weighted MR images of the brain with contrast enhancement demonstrating a homogeneously enhancing, right posterior fossa extraaxial lesion with extension into the sigmoid sinus. Preoperative axial CT angiogram (C) of the brain showing partial occlusion of the right sigmoid sinus due to tumor compression and invasion. 

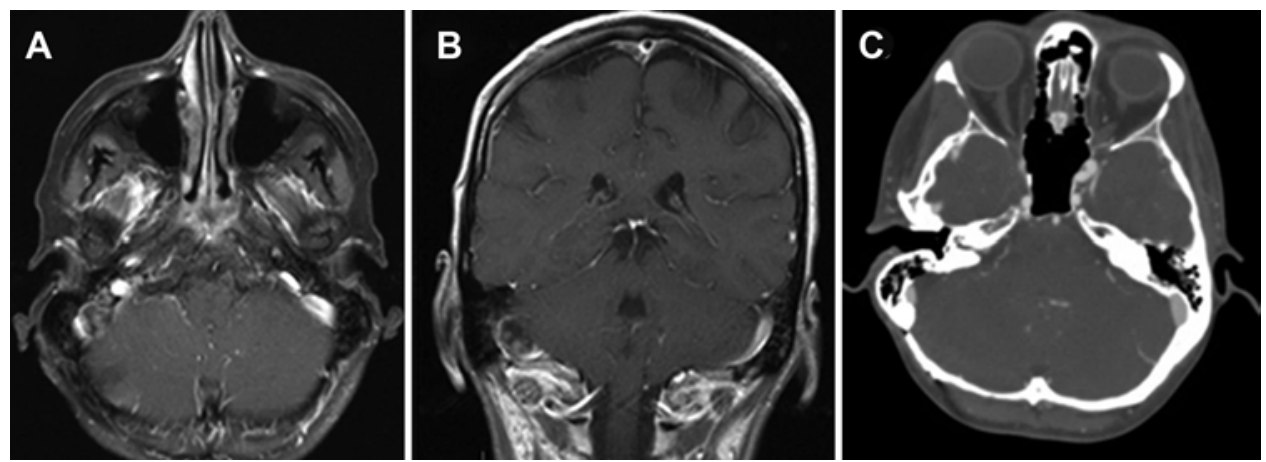

FIG. 2. Case 1. Postoperative axial (A) and coronal (B) T1-weighted MR images of the brain with contrast enhancement showing GTR of the right posterior fossa meningioma. Postoperative axial CT angiogram (C) of the brain demonstrating patency of the right sigmoid sinus.

occipital craniotomy was performed. Surgical exploration revealed that the inferior aspect of the tumor had invaded the wall of the transverse sinus. After the tumor and the involved sinus wall were completely resected, the sinus was reconstructed using a small piece of allogeneic tissue graft (AlloDerm, LifeCell Corp.). The patient received $600 \mathrm{mg}$ of aspirin rectally along with 1000 units of intravenous heparin intraoperatively to prevent sinus thrombosis after the repair. He was maintained on $81 \mathrm{mg}$ of aspirin daily throughout his postoperative hospitalization. Pathological analysis was consistent with a WHO Grade I meningioma. Magnetic resonance imaging on postoperative Day 1 demonstrated gross-total tumor resection, while a CT venogram revealed partial occlusion
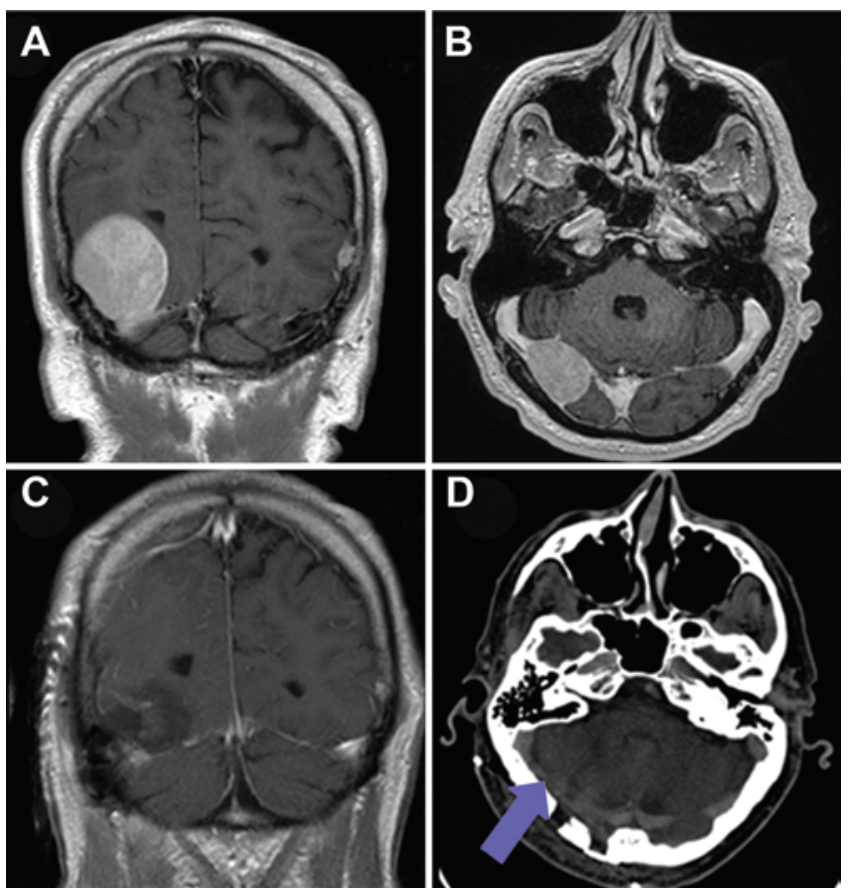

FIG. 3. Case 2. Preoperative coronal (A) and axial (B) T1-weighted MR images of the brain with contrast enhancement demonstrating a homogeneously enhancing, right extraaxial tentorial lesion with invasion of the transverse sinus. Postoperative coronal T1-weighted MR image (C) of the brain with contrast enhancement demonstrating GTR. Postoperative axial CT venogram (D) of the brain showing partial thrombosis (arrow) of the right transverse sinus. of the transverse sinus in the repaired segment with adequate reconstitution distally (Fig. 3C and D). Although the patient remained clinically stable, he was started on full anticoagulation therapy to prevent further clot propagation before being discharged home on postoperative Day 3.

\section{Case 3}

A 76-year-old woman with progressive gait difficulty was diagnosed with a $4 \times 3 \times 4-\mathrm{cm}$ homogeneously enhancing extraaxial lesion in the left posterior fossa with involvement of the transverse sinus (Fig. 4A and B). She
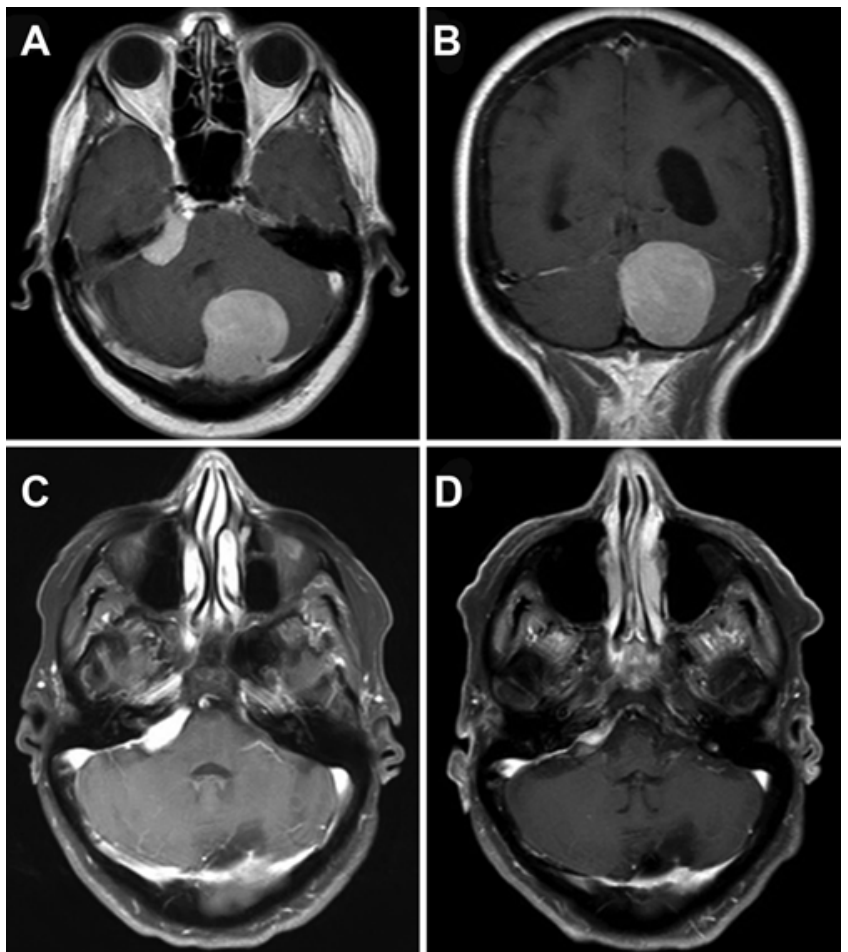

FIG. 4. Case 3. Preoperative axial (A) and coronal (B) T1-weighted MR images of the brain with contrast enhancement demonstrating a homogeneously enhancing, left posterior fossa extraaxial lesion with involvement of the transverse sinus. Immediately postoperative $(\mathrm{C})$ and 2-year follow-up (D) axial T1-weighted MR images of the brain with contrast enhancement demonstrating no progression of a small residual tumor around the transverse sinus. 


\section{Meningiomas of the transverse or sigmoid sinuses}
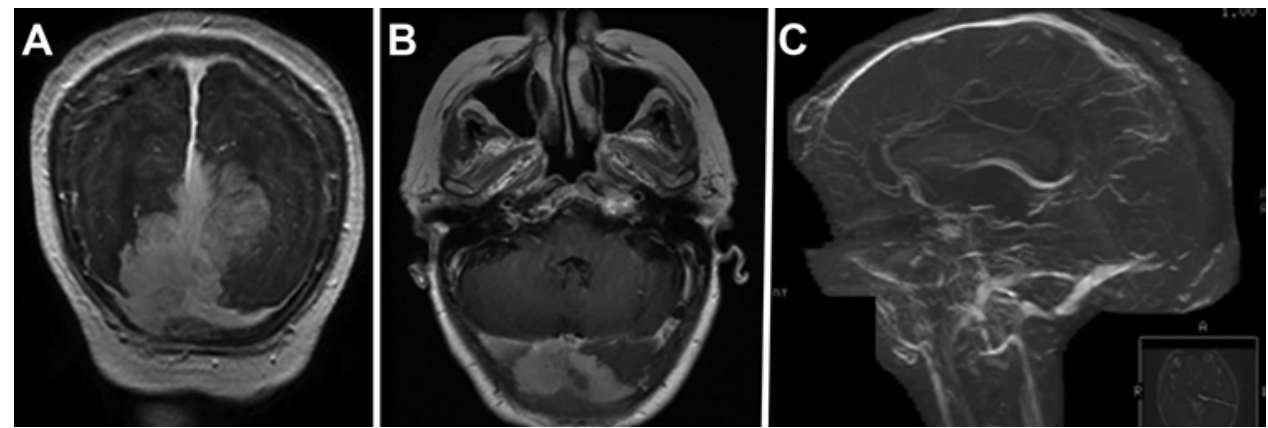

FIG. 5. Case 4. Preoperative coronal (A) and axial (B) T1-weighted MR images of the brain with contrast enhancement demonstrating a homogeneously enhancing extraaxial lesion in the bilateral occipital region with involvement of the posterior third of the superior sagittal sinus, the confluence of sinuses, and the bilateral transverse sinuses. Magnetic resonance angiogram (C) of the brain demonstrating complete occlusion of the sinuses in the region of tumor involvement with collateral venous drainage.

underwent a suboccipital craniectomy for tumor resection. Intraoperatively, there was extensive tumor invasion through the transverse sinus wall; however, considering the patient's advanced age and risk of hemorrhage, it was decided not to proceed with an aggressive intrasinus tumor resection. The tumor was debulked, and the dura was cauterized to the outer sinus wall, but a small portion of residual tumor remained involving the transverse sinus. Pathological analysis of the tumor was consistent with a WHO Grade I meningioma. Follow-up imaging at 2 years after resection demonstrated no significant enlargement of residual tumor within the region of the transverse sinus (Fig. 4C and D).

\section{Case 4}

A 78-year-old woman presented with progressive visual decline and difficulty walking. Imaging of the brain revealed a diffuse, homogeneously enhancing extraaxial mass in the bilateral occipital region with evidence of bone invasion and involvement of the posterior third of the superior sagittal sinus, the confluence of sinuses, and the bilateral transverse sinuses. Vascular imaging confirmed occlusion of these sinuses within this region (Fig. 5). Intraoperatively, the posterior superior sagittal and bilateral transverse sinuses were ligated just beyond the margins of tumor invasion, allowing for a near-complete tumor resection. Pathological analysis of the tissue was
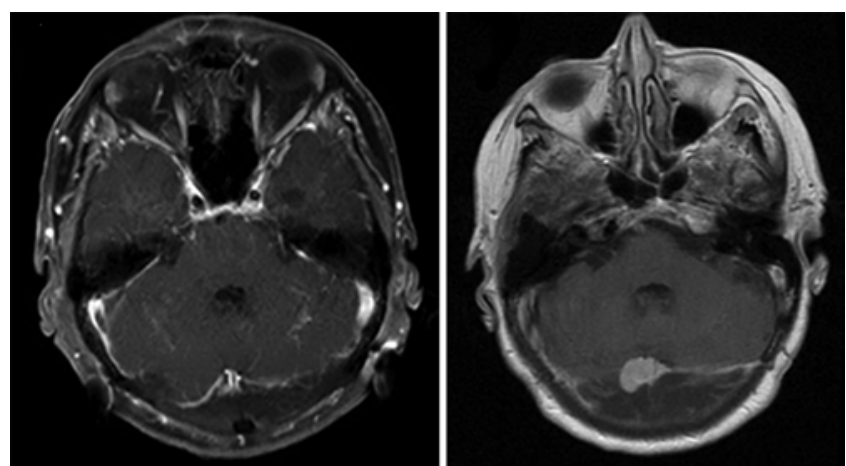

FIG. 6. Case 4. Immediately postoperative (left) and 2-year followup (right) axial T1-weighted MR images of the brain with contrast enhancement demonstrating new tumor growth in the region of the right transverse sinus. The new growth was subsequently treated with stereotactic radiosurgery. consistent with a WHO Grade I meningioma. Postoperative imaging revealed no residual tumor. At the 2-year follow-up, however, a small tumor recurrence was noted along the margin on the right transverse sinus (Fig. 6). The patient underwent stereotactic radiosurgery treatment for the lesion, which at 1 year postradiation demonstrated no further progression. In addition, no radiation treatment-related side effects have occurred.

\section{Conclusions}

The management of meningiomas of the transverse or sigmoid sinuses often varies on a case-by-case basis. Depending on the degree of tumor invasion, sinus patency, surgeon experience, patient age, and tumor histology, the spectrum of treatments may range from radical resection with sinus reconstruction to subtotal resection followed by close monitoring with radiation therapy for recurrent disease. The few studies in the literature that focus on meningiomas in this location suggest that both aggressive and conservative treatment strategies can be successful in certain patient populations. Studies reporting long-term outcomes, however, are lacking. We provide illustrative cases to demonstrate the clinical scenarios in which various treatment strategies can be applied.

\section{Acknowledgment}

We thank Kristin Kraus, M.Sc., for editorial assistance in the preparation of this paper.

\section{Disclosure}

The authors report no conflict of interest concerning the materials or methods used in this study or the findings specified in this paper.

Author contributions to the study and manuscript preparation include the following. Conception and design: Mazur, Taussky. Acquisition of data: Mazur, Cutler. Analysis and interpretation of data: Mazur, Cutler. Drafting the article: Mazur, Cutler. Critically revising the article: all authors. Reviewed submitted version of manuscript: all authors. Study supervision: Couldwell, Taussky.

\section{References}

1. Aghi MK, Carter BS, Cosgrove GR, Ojemann RG, AminHanjani S, Martuza RL, et al: Long-term recurrence rates of 
atypical meningiomas after gross total resection with or without postoperative adjuvant radiation. Neurosurgery 64:5660, 2009

2. Arnautović KI, Al-Mefty O, Angtuaco E, Phares LJ: Dural arteriovenous malformations of the transverse/sigmoid sinus acquired from dominant sinus occlusion by a tumor: report of two cases. Neurosurgery 42:383-388, 1998

3. Bassiouni H, Hunold A, Asgari S, Stolke D: Tentorial meningiomas: clinical results in 81 patients treated microsurgically. Neurosurgery 55:108-118, 2004

4. Bonnal J, Brotchi J: Surgery of the superior sagittal sinus in parasagittal meningiomas. J Neurosurg 48:935-945, 1978

5. Caroli E, Orlando ER, Mastronardi L, Ferrante L: Meningiomas infiltrating the superior sagittal sinus: surgical considerations of 328 cases. Neurosurg Rev 29:236-241, 2006

6. Castellano F, Ruggiero G: Meningiomas of the posterior fossa. Acta Radiol Suppl 104:1-177, 1953

7. Chausson N, Bocquet J, Aveillan M, Olindo S, Signaté A, Merle H, et al: Intracranial hypertension caused by a meningioma compressing the transverse sinus. J Clin Neurosci 17: 1589-1592, 2010

8. Colli BO, Assirati JA Jr, Deriggi DJP, Neder L, dos Santos AC, Carlotti CG Jr: Tentorial meningiomas: follow-up review. Neurosurg Rev 31:421-430, 2008

9. Condra KS, Buatti JM, Mendenhall WM, Friedman WA, Marcus RB Jr, Rhoton AL: Benign meningiomas: primary treatment selection affects survival. Int J Radiat Oncol Biol Phys 39:427-436, 1997

10. Conti A, Pontoriero A, Salamone I, Siragusa C, Midili F, La Torre D, et al: Protecting venous structures during radiosurgery for parasagittal meningiomas. Technical note. Neurosurg Focus 27(5):E11, 2009

11. Damak M, Crassard I, Wolff V, Bousser MG: Isolated lateral sinus thrombosis: a series of 62 patients. Stroke 40:476-481, 2009

12. Day JD, Fukushima T, Giannotta SL: Cranial base approaches to posterior circulation aneurysms. J Neurosurg 87:544-554, 1997

13. DiMeco F, Li KW, Casali C, Ciceri E, Giombini S, Filippini G, et al: Meningiomas invading the superior sagittal sinus: surgical experience in 108 cases. Neurosurgery 55:1263-1274, 2004

14. Ganesan D, Higgins JNP, Harrower T, Burnet NG, Sarkies NJC, Manford M, et al: Stent placement for management of a small parasagittal meningioma. Technical note. J Neurosurg 108:377-381, 2008

15. Giombini S, Solero CL, Lasio G, Morello G: Immediate and late outcome of operations for parasagittal and falx meningiomas. Report of 342 cases. Surg Neurol 21:427-435, 1984

16. Goyal LK, Suh JH, Mohan DS, Prayson RA, Lee J, Barnett GH: Local control and overall survival in atypical meningioma: a retrospective study. Int J Radiat Oncol Biol Phys 46:57-61, 2000

17. Guclu B, Sindou M: Reconstruction of vein of Labbé in temporo-occipital meningioma invading transverse sinus: technical report. Acta Neurochir (Wien) 152:941-945, 2010

18. Harris AE, Lee JY, Omalu B, Flickinger JC, Kondziolka D, Lunsford LD: The effect of radiosurgery during management of aggressive meningiomas. Surg Neurol 60:298-305, 2003

19. Heros RC: Editorial. Simpson Grade and MIB-1. J Neurosurg 117:118-120, 2012

20. Heros RC: Editorial. Simpson grades. J Neurosurg 117:997998, 2012

21. Hoessly GF, Olivecrona H: Report on 280 cases of verified parasagittal meningioma. J Neurosurg 12:614-626, 1955

22. Hug EB, Devries A, Thornton AF, Munzenride JE, Pardo FS, Hedley-Whyte ET, et al: Management of atypical and malignant meningiomas: role of high-dose, 3D-conformal radiation therapy. J Neurooncol 48:151-160, 2000

23. Hwang SK, Gwak HS, Paek SH, Kim DG, Jung HW: Guide- lines for the ligation of the sigmoid or transverse sinus during large petroclival meningioma surgery. Skull Base 14:21-29, 2004

24. Inamura T, Nishio S, Takeshita I, Fujiwara S, Fukui M: Peritumoral brain edema in meningiomas - influence of vascular supply on its development. Neurosurgery 31:179-185, 1992

25. Kim AW, Trobe JD: Syndrome simulating pseudotumor cerebri caused by partial transverse venous sinus obstruction in metastatic prostate cancer. Am J Ophthalmol 129:254-256, 2000

26. Kondziolka D, Flickinger JC, Perez B: Judicious resection and/or radiosurgery for parasagittal meningiomas: outcomes from a multicenter review. Neurosurgery 43:405-414, 1998

27. Kondziolka D, Mathieu D, Lunsford LD, Martin JJ, Madhok R, Niranjan A, et al: Radiosurgery as definitive management of intracranial meningiomas. Neurosurgery 62:53-60, 2008

28. Maiuri F, Di Martino G, Vergara P, Mariniello G: Meningiomas of the transverse - sigmoid sinus junction area. Br J Neurosurg 25:492-496, 2011

29. Mantovani A, Di Maio S, Ferreira M Jr, Sekhar LN: Management of meningiomas invading the major dural venous sinuses: operative technique, results, and potential benefit for higher grade tumors. World Neurosurg [epub ahead of print], 2013

30. Owler BK, Parker G, Halmagyi GM, Dunne VG, Grinnell V, McDowell D, et al: Pseudotumor cerebri syndrome: venous sinus obstruction and its treatment with stent placement. J Neurosurg 98:1045-1055, 2003

31. Oya S, Kawai K, Nakatomi H, Saito N: Significance of Simpson grading system in modern meningioma surgery: integration of the grade with MIB-1 labeling index as a key to predict the recurrence of WHO Grade I meningiomas. Clinical article. J Neurosurg 117:121-128, 2012

32. Pearson BE, Markert JM, Fisher WS, Guthrie BL, Fiveash JB, Palmer CA, et al: Hitting a moving target: evolution of a treatment paradigm for atypical meningiomas amid changing diagnostic criteria. A case series. Neurosurg Focus 24(5):E3, 2008

33. Pettersson-Segerlind J, Orrego A, Lönn S, Mathiesen T: Longterm 25-year follow-up of surgically treated parasagittal meningiomas. World Neurosurg 76:564-571, 2011

34. Pollock BE, Stafford SL, Utter A, Giannini C, Schreiner SA: Stereotactic radiosurgery provides equivalent tumor control to Simpson Grade 1 resection for patients with small- to medium-size meningiomas. Int J Radiat Oncol Biol Phys 55:1000-1005, 2003

35. Raza SM, Gallia GL, Brem H, Weingart JD, Long DM, Olivi A: Perioperative and long-term outcomes from the management of parasagittal meningiomas invading the superior sagittal sinus. Neurosurgery 67:885-893, 2010

36. Rogers L, Gilbert M, Vogelbaum MA: Intracranial meningiomas of atypical (WHO grade II) histology. J Neurooncol 99:393-405, 2010

37. Schmid-Elsaesser R, Steiger HJ, Yousry T, Seelos KC, Reulen HJ: Radical resection of meningiomas and arteriovenous fistulas involving critical dural sinus segments: experience with intraoperative sinus pressure monitoring and elective sinus reconstruction in 10 patients. Neurosurgery 41:1005-1018, 1997

38. Sekhar LN, Chanda A, Morita A: The preservation and reconstruction of cerebral veins and sinuses. J Clin Neurosci 9: 391-399, 2002

39. Sekhar LN, Tzortzidis FN, Bejjani GK, Schessel DA: Saphenous vein graft bypass of the sigmoid sinus and jugular bulb during the removal of glomus jugulare tumors. Report of two cases. J Neurosurg 86:1036-1041, 1997

40. Simpson D: The recurrence of intracranial meningiomas after surgical treatment. J Neurol Neurosurg Psychiatry 20:2239, 1957 


\section{Meningiomas of the transverse or sigmoid sinuses}

41. Sindou M: Meningiomas invading the sagittal or transverse sinuses, resection with venous reconstruction. J Clin Neurosci 8 Suppl 1:8-11, 2001

42. Sindou M, Auque J, Jouanneau E: Neurosurgery and the intracranial venous system. Acta Neurochir Suppl 94:167-175, 2005

43. Sindou MP, Alvernia JE: Results of attempted radical tumor removal and venous repair in 100 consecutive meningiomas involving the major dural sinuses. J Neurosurg 105:514-525, 2006

44. Singh VP, Kansai S, Vaishya S, Julka PK, Mehta VS: Early complications following gamma knife radiosurgery for intracranial meningiomas. J Neurosurg 93 (Suppl 3):57-61, 2000

45. Spetzler RF, Daspit CP, Pappas CT: The combined supra- and infratentorial approach for lesions of the petrous and clival regions: experience with 46 cases. J Neurosurg 76:588-599, 1992

46. Sughrue ME, Kane AJ, Shangari G, Rutkowski MJ, McDermott MW, Berger MS, et al: The relevance of Simpson Grade I and II resection in modern neurosurgical treatment of World Health Organization Grade I meningiomas. Clinical article. J Neurosurg 113:1029-1035, 2010

47. Sughrue ME, Rutkowski MJ, Shangari G, Parsa AT, Berger MS, McDermott MW: Results with judicious modern neurosurgical management of parasagittal and falcine meningiomas. Clinical article. J Neurosurg 114:731-737, 2011
48. Tanaka M, Imhof HG, Schucknecht B, Kollias S, Yonekawa Y, Valavanis A: Correlation between the efferent venous drainage of the tumor and peritumoral edema in intracranial meningiomas: superselective angiographic analysis of 25 cases. J Neurosurg 104:382-388, 2006

49. Tomasello F, Conti A, Cardali S, Angileri FF: Venous preservation-guided resection: a changing paradigm in parasagittal meningioma surgery. Clinical article. J Neurosurg 119:7481,2013

50. Yokota M, Tani E, Maeda Y, Yamaura I: Meningioma in sigmoid sinus groove associated with dural arteriovenous malformation: case report. Neurosurgery 33:316-319, 1993

51. Zilani G, Pereira EA, Baig F, Uberoi R, Kuker W, Kerr RS: Venoplasty and stenting of a jugular foramen meningioma. $\mathbf{B r}$ J Neurosurg 23:557-560, 2009

Manuscript submitted August 15, 2013.

Accepted August 29, 2013.

Please include this information when citing this paper: DOI: 10.3171/2013.8.FOCUS13340.

Address correspondence to: Philipp Taussky, M.D., Department of Neurosurgery, University of Utah, 175 N. Medical Drive E., Salt Lake City, UT 84132. email: neuropub@hsc.utah.edu. 